\title{
Criptococose palpebral: relato de caso
}

\author{
Palpebralcryptococcosis:case report
}

\author{
Murilo BarretoSouza ${ }^{1}$ \\ Carlos Sergio Nascimento Melo \\ Cristiana Silveira Silva ${ }^{3}$ \\ Ruth MiyukiSanto ${ }^{4}$ \\ Suzana Matayoshi ${ }^{5}$
}

\section{RESUMO}

Relata-se o caso de uma paciente portadora de síndrome da imunodeficiência adquirida empiricamente tratada com esquema tríplice para tuberculose miliar. Durante a evolução clínica a paciente cursou com lesões comprometendo a pálpebra e conjuntiva tarsal à direita. A hipótese diagnóstica inicial foi de tuberculose ocular com comprometimento conjuntival e palpebral. A biópsia da lesão conjuntival mostrou presença do Criptococcus neoformans. Após o início do tratamento específico com anfotericina B, a paciente apresentou melhora das lesões cutâneas.

Descritores: Infecções oportunistas relacionadas com a AIDS; Criptococose/microbiologia; Cryptococcus neoformans; Doenças palpebrais/microbiologia; Infecções oculares fúngicas; Anfotericina B/uso terapêutico; Relatos de casos [tipo de publicação]

\section{INTRODUÇÃO}

A criptococose é uma infecção oportunística, causada pelo Cryptococcus neoformans, um fungo dimorfo e encapsulado, de distribuição univer$\mathrm{sal}^{(1)}$. A infecção primária em indivíduos sadios é possível, porém rara, ocorrendo com maior freqüência em indivíduos imunodeprimidos, quando se apresenta como uma doença grave e potencialmente fatal. O sítio inicial da infecção é geralmente o pulmão, onde a infecção pode permanecer de forma latente ou oligossintomática por um longo período. Em 10\% dos casos evolui com disseminação hematogênica, com predileção especial pelo sistema nervoso central ${ }^{(1)}$. O comprometimento ósseo, cutâneo ou ocular é bem menos freqüente ${ }^{(2)}$. É descrito a seguir o caso de uma paciente portadora da síndrome da imunodeficiência adquirida (SIDA), com comprometimento palpebral e conjuntival pelo $C$. neoformans.

${ }^{1}$ Médico oftalmologista. Docente da Disciplina de Informática Médica da Faculdade de Medicina da Faculdade de Ciência e Tecnologia - FTC - Salvador (BA) - Brasil. ${ }^{2}$ Fellow do Serviço de Retina e Vítreo do Hospital das Clínicas da Faculdade de Medicina da Universidade de São Paulo - USP - São Paulo (SP) - Brasil.

${ }^{3}$ Médica Dermatologista.

${ }^{4}$ Doutora em oftalmologia pela USP - São Paulo (SP) Brasil. Médica Assistente do Hospital das Clínicas da Faculdade de Medicina da USP - São Paulo (SP) - Brasil. ${ }^{5}$ Doutora em oftalmologia pela USP - São Paulo (SP) Brasil. Médica Assistente do Hospital das Clínicas da Faculdade de Medicina da USP - São Paulo (SP) - Brasil. Endereço para correspondência: Murilo Barreto Souza. Rua Alberto Ponde, 109/503 - Salvador (BA) CEP 40280-690

E-mail: murilobarreto@ig.com.br

Recebido para publicação em 11.12.2003

Versão revisada recebida em 24.11.2005

Aprovação em 02.12.2005

\section{RELATO DE CASO}

EMS, sexo feminino, 43 anos, negra, natural e procedente de São Paulo, com diagnóstico de SIDA há 7 meses, em uso de terapia anti-retroviral desde então. A paciente apresentava quadro clínico compatível com tuberculose miliar há 4 meses, encontrando-se em uso empírico do esquema tríplice há 3 meses, tendo cursado com discreta melhora do estado geral. Evoluiu com aparecimento de edema em pálpebra superior direita, acompanhado de linfadenopatia cervical e lesões cutâneas na face. Negava secreção ocular ou baixa de acuidade visual.

Ao exame clínico apresentava acuidade visual corrigida de 20/20 em ambos os olhos, motricidade ocular extrínseca, pressão intra-ocular e reflexo fotomotor sem alterações. Exibia edema de terço lateral da pálpebra superior direita acompanhado de nódulo de consistência fibroelástica, e úlcera com bordas elevadas, recoberto por material sero-hemático, associa-
\end{abstract}


dos a pápulas, algumas agrupadas, tendendo a umbilicação central, na pele da região temporal (Figura 1). À biomicroscopia apresentava perda de cílios na borda lateral da pálpebra superior direita, e tumoração envolvendo a mesma região com comprometimento da conjuntiva tarsal (Figura 2). Não foram observadas alterações fundoscópicas. O olho esquerdo era normal. Devido à suspeita clínica inicial de tuberculose miliar, foi feita a hipótese diagnóstica de tuberculose palpebral e

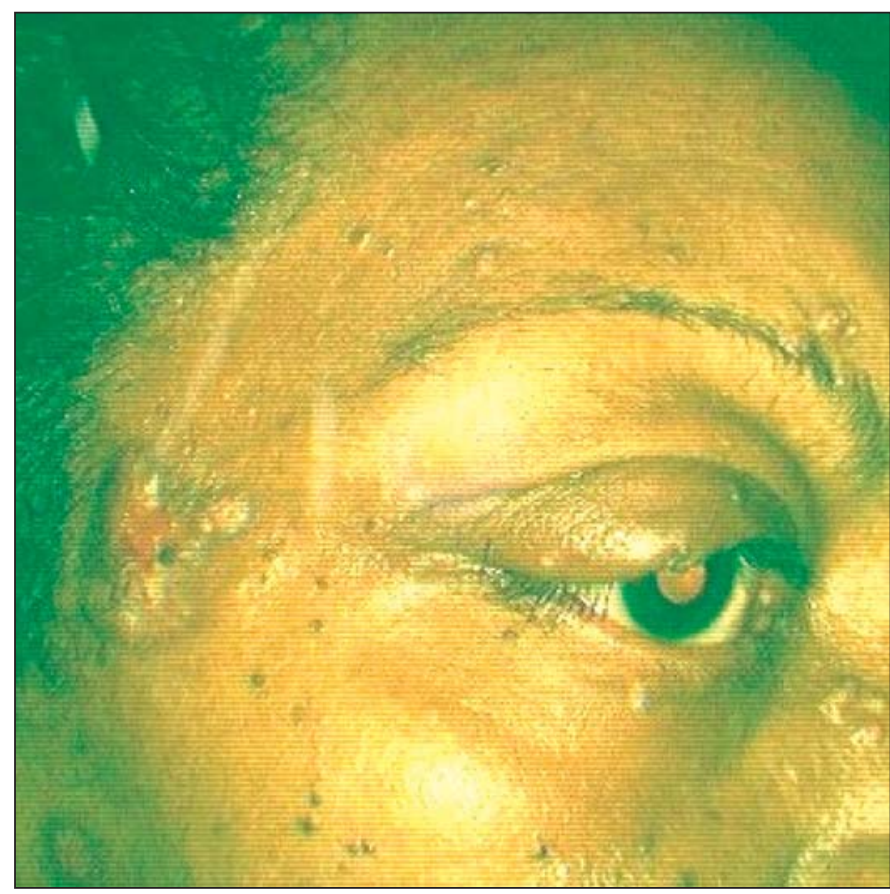

Figura 1 - Edema de terço lateral da pálpebra superior direita acompanhado de úlcera com bordos elevados, recoberto por material serohemático, e pápulas na face

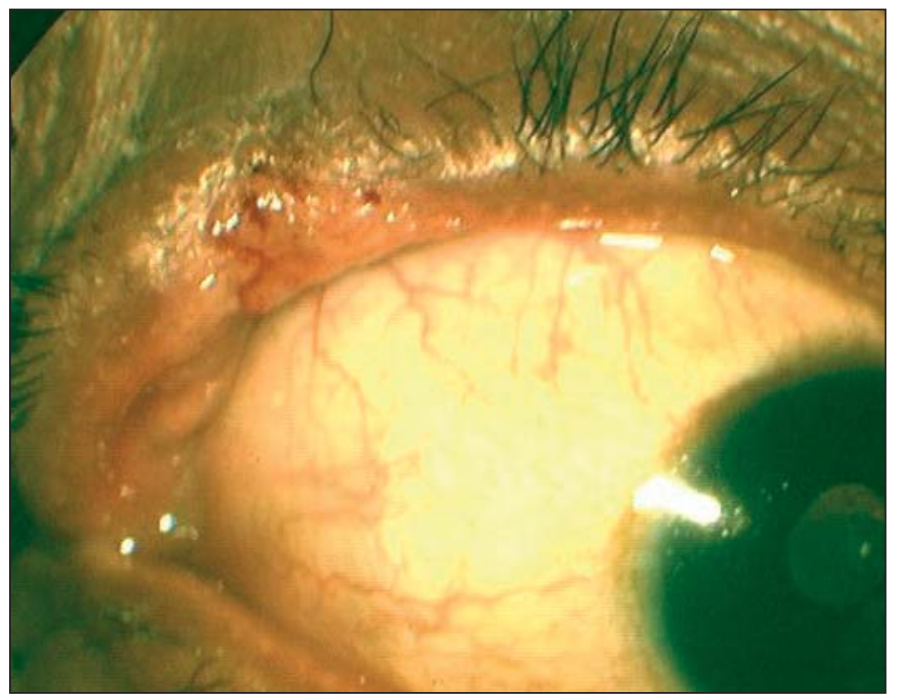

Figura 2 - Perda de cílios em borda lateral da pálpebra superior direita, e tumoração envolvendo a mesma região com comprometimento da conjuntiva tarsal indicada a realização de biópsia da lesão conjuntival. O exame anátomo-patológico evidenciou presença de histiócitos e células gigantes multinucleadas após coloração pela hematoxilina-eosina. A pesquisa de bacilo álcool-ácido resistente pela coloração de Ziehl-Nielsen foi negativa. A coloração com o mucicarmim evidenciou a presença de blastoconídeos com cápsula radiada, compatíveis com C. neoformans (Figura 3). Foi iniciado o tratamento com anfotericina B, $1 \mathrm{mg} / \mathrm{kg} / \mathrm{d}$, seguido de fluconazol $400 \mathrm{mg} / \mathrm{d}$. A paciente evoluiu com melhora das lesões 3 semanas após início do tratamento, e permaneceu assintomática durante 7 meses, voltando a apresentar novas lesões cutâneas na face, 1 mês após interrupção do tratamento, quando foi reiniciado o uso de fluconazol.

\section{DISCUSSÃO}

A criptococose é uma micose rara e potencialmente fatal, que acomete mais freqüentemente indivíduos adultos imunodeprimidos $^{(1)}$. Desde o surgimento da síndrome da imunodeficiência adquirida a criptococose vem se tornando cada vez mais freqüente, atingindo 5 a $10 \%$ destes pacientes ao longo da vida, principalmente na forma de acometimento pulmonar, representando a principal causa de infecção fúngica grave ${ }^{(3)}$. As manifestações cutâneas são observadas em 10 a 20\% dos pacientes, e se apresentam clinicamente de várias formas, acometendo principalmente a cabeça e o pescoço ${ }^{(2)}$. Em pacientes portadores de SIDA, as lesões cutâneas da criptococose podem apresentar umbilicação central simulando o molusco contagioso, como aconteceu em algumas lesões cutâneas neste caso. A manifestação ocular mais freqüente é o envolvimento do segmento posterior na forma de atrofia óptica, papiledema, endoftalmite e coroidite ${ }^{(3)}$. Há também descrições de envolvimento iriano $^{(4)}$, da região límbica ${ }^{(5)}$, e comprometimento palpebral ${ }^{(2)} \mathrm{e}$ conjuntival ${ }^{(3)}$, como o relatado neste caso. Não há relato específico de comprometimento palpebral isoladamente.

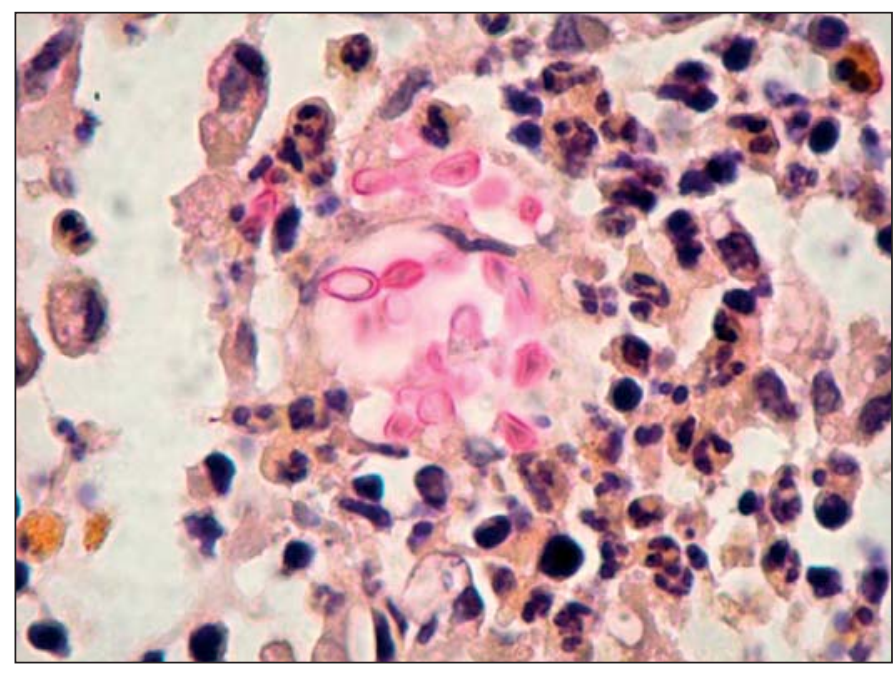

Figura 3 - Corte histológico corado pelo mucicarmim evidenciando blastoconídeo com cápsula radiada $(40 x)$ 
O prognóstico depende do grau de comprometimento imunológico da paciente, da magnitude e sítios de comprometimento da infecção pelo $C$. neoformans, e do início imediato do tratamento $^{(2)}$. Neste caso observamos a importância do exame anátomo-patológico, uma vez que, na ausência do isolamento do Mycobacterium tuberculosis, a paciente foi empiricamente tratada para tuberculose miliar por 3 meses sem melhora satisfatória do quadro. Com a identificação de um agente etiológico, através da biópsia palpebral e conjuntival, compatível com as lesões estudadas e com o quadro clínico sistêmico, e após iniciado o tratamento específico, a paciente apresentou resolução das lesões da face, pálpebra e conjuntiva, e melhora acentuada do quadro sistêmico. Entre os achados histopatológicos que auxiliam no diagnóstico da criptococose, a identificação da cápsula radiada torna-se mais difícil em pacientes imunossuprimidos, uma vez que esta é formada pela resposta imune do hospedeiro ao parasita ${ }^{(1)}$. A recidiva do quadro após interrupção do tratamento, como observado neste caso, não é rara em pacientes portadores de SIDA.

\section{ABSTRACT}

This paper is about a patient with acquired immunodeficiency syndrome empirically treated for miliary tuberculosis. During the clinical evolution the patient presented lesions compromi- sing the right eyelid and tarsal conjunctiva. The initial diagnostic hypothesis was ocular tuberculosis with conjunctival and eyelid involvement. The biopsy of the conjuctival lesion identified an encapsulated yeast-like fungus: Criptococcus neoformans. After starting treatment with B anfotericin, the cutaneus lesions cleared.

Keywords: AIDS-related opportunistic infections; Cryptococcosis/microbiology; Cryptococcus neoformans; Eyelid diseases/microbiology; Eye infections, fungal; Amphotericin B/ therapeutic use; Case reports [publication type]

\section{REFERÊNCIAS}

1. Lacaz CS, Porto E, Martins JEC, Heins-Vavvari EM, Melo NT. Criptococose. In: Lacaz CS, Porto E, Martins JEC, Vaccari EMH, Melo NT, editores. Tratado de micologia médica Lacaz. $9^{a}$ ed. São Paulo: Sarvier; 2002. p.416-40.

2. Coccia L, Calista D, Boschini A. Eyelid nodule: a sentinel lesion of disseminated cryptococcosis in a patient with acquired immunodeficiency syndrome. Arch Ophthalmol. 1999;117(2):271-2.

3. Kestelyn P, Taelman H, Bogaerts J, Kagame A, Abdel Aziz M, Batungwanayo $\mathrm{J}$, et al. Ophthalmic manifestations of infections with Cryptococcus neoformans in patients with the acquired immunodeficiency syndrome. Am J Ophthalmol. 1993;116(6):721-7.

4. Charles NC, Boxrud CA, Smal EA. Cryptococcosis of the anterior segment in acquired immune deficiency syndrome. Ophthalmology. 1992;99(5):813-6.

5. Muccioli C, Belfort Junior R, Neves R, Rao N. Limbal and choroidal Cryptococcus infection in the acquired immunodeficiency syndrome. Am J Ophthalmol. 1995;122(4):539-40.

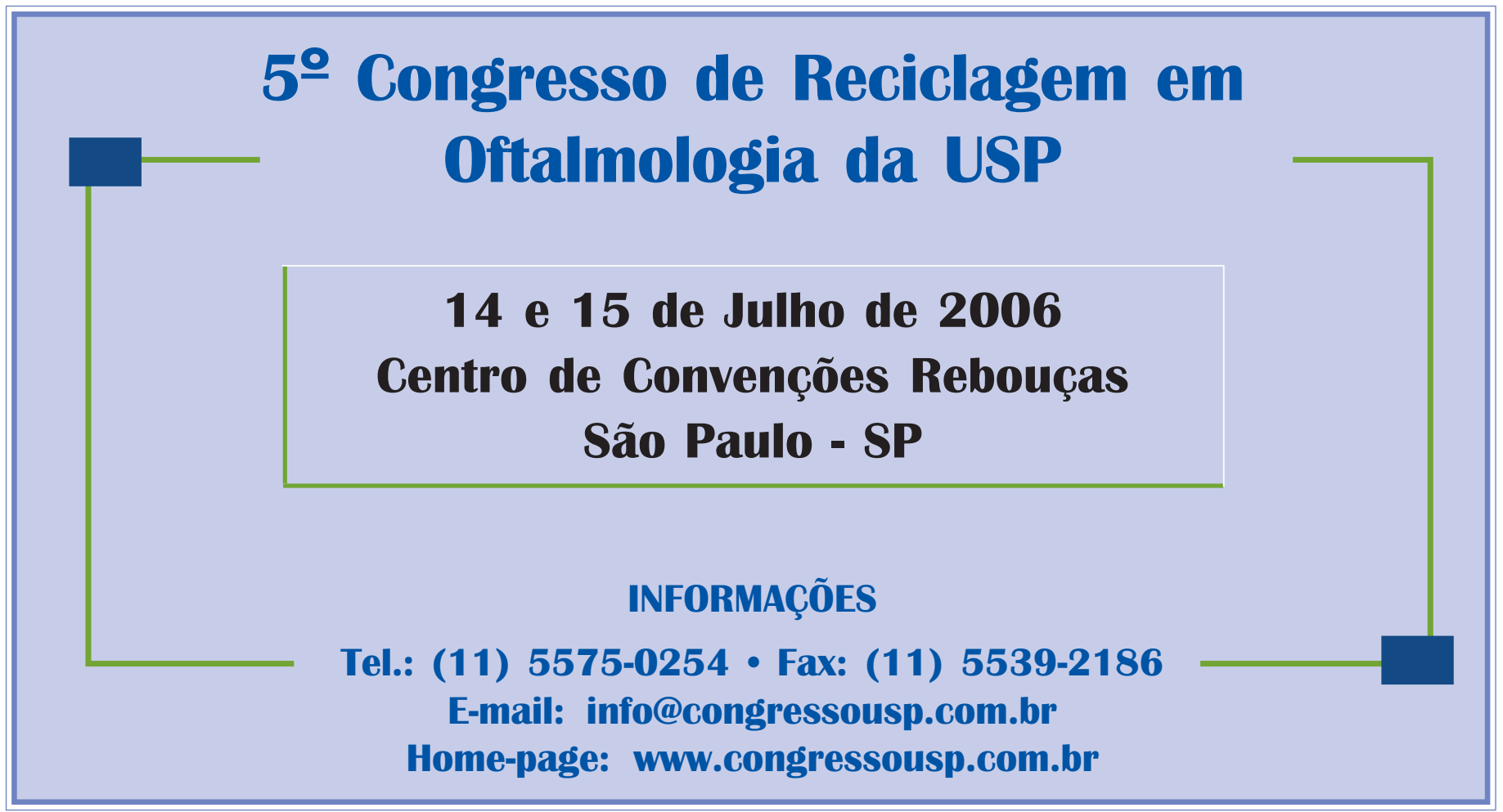

